
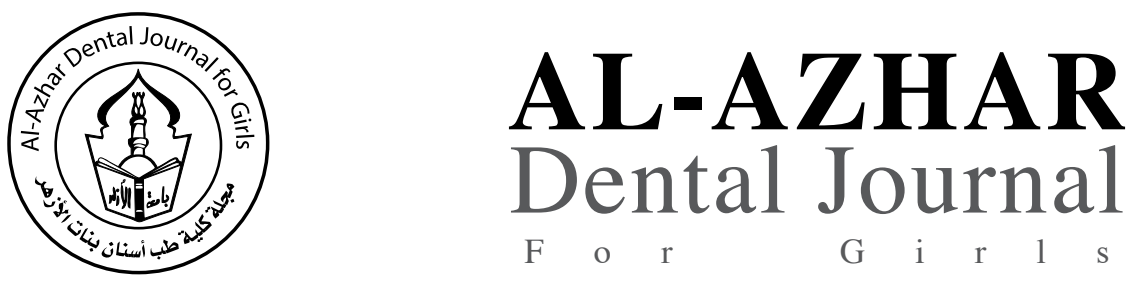

The Official Publication of The Faculty of Dental Medicine For Girls, Al-Azhar University Cairo, Egypt.

ADJ-for Grils, Vol. 4, No. 3, July (2017) - PP. 223:229

\title{
Effect of Natural Cross-Linkers on Shear Bond Strength to Dentin After Storage in Different Media
}

\section{Kamal Eldeen A. Elmobsher ${ }^{(1)}$ Maha A. Niazy $^{(2)}$, Inas T. Motawea ${ }^{(3)}$ and Mayada S. Sultan $^{(4)}$}

Codex : 27/1707

dentaljournal.forgirls@yahoo.com

\begin{abstract}
Objectives: This study was designed to evaluate the effect of natural cross linkers (Propolis and Hesperidin) on shear bond strength (SBS) to dentin after storage in different media. Materials and Methods: Ninety extracted molars were used and divided into three main groups according to dentin surface treatment $(n=30)$ where, A1: treated with Propolis, A2: treated with Hesperidin and A3: receive no treatment to serve as a control group. Each group was further subdivided into 3 subgroups $(n=10)$ according to the storage media either stored in Acids (B1) or stored in Artificial saliva (B2) or stored in distilled Water (B3). The shear bond strength (SBS) was determined using a universal testing machine. Results: The dentin specimens treated with Propolis showed significantly highest SBS. Conclusion: Both Propolis and Hesperidin cross linking agents are considered beneficial in improving bonding to dentin, though Propolis was much more effective. Storage media has deteriorating effect on resin-dentin shear bond strength.
\end{abstract}

\section{INTRODUCTION}

The achievement of an efficient and stable bond between tooth substrate and resin materials remains a challenge in restorative dentistry. The primary aim of dental adhesives is to provide retention to composite cements and withstand mechanical forces and shrinkage stress from the lining composite ${ }^{(1)}$. Clinically, failure of restorations occurs mainly due to inadequate sealing and hydrolytic degradation of both

Collagen cross-linkers,

Propolis, Hesperidin,

Shear bond strength

Paper extracted from Master thesis entitled: "Effect of Natural Cross-Linkers on Nanoleakage and Shear Bond Strength to Dentin After Storage in Different Media"

1. Dentist at Ministry of Health, Cairo, Egypt.

2. Professor and Head of Operative Dentistry Department, Faculty of Dental Medicine for girlsAl Azhar University.

3. Assistant Professor and Head of Dental Biomaterials Department, Faculty of Dental Medicine for girls Al Azhar University.

4. Lecture of Operative Dentistry, Faculty of Dental Medicine, Assuit University. 
resin material and dentin collagen that leads to loss of retention ${ }^{(2,3)}$.

Most of the developments in adhesive dentistry have focused on the improvement of bonding agents and techniques, while limited investigations have explored the contributions of collagen structure and stability to the bond strength. The presence of cross-linkers in solubilized collagen provide strength, reinforcement and stabilization of fibrils ${ }^{(4)}$. Extrinsic collagen cross-linking agents may induce additional inter- and intra-molecular cross-links, enhancing collagen mechanical properties and its resistance to enzymatic degradation which is advantageous to the dentin bonding ${ }^{(5)}$.

Propolis is a natural resinous material that honeybees collect from various plant species. Flavanoids in propolis, being natural anti-oxidants and free radical scavengers, increase collagen synthesis and enhance collagen stabilization ${ }^{(6)}$.

Hesperidin is a glycoside flavonoid antioxidant abundantly present in citrus fruits, The alleged action of this natural agent in protecting collagen against degradation is inhibition of MMPs and stabilization of the collagenous matrix ${ }^{(7)}$.

Storage media is an important factor that affects the durability of the bond. Period of immersion in solutions as well as the frequency that this solution is changed can be important for degradation process $^{(8)}$. Therefore, this study was designed to evaluate the effect of collagen cross linkers on bond strength to dentin.

\section{MATERIALS AND METHODS}

\section{Teeth Selection:}

A total of ninety human sound molars were collected for this study and extracted from an age group of 20-40 years due to impaction or periodontal reasons to obtain specimens of sound dentin. Immediately after extraction, each tooth was thoroughly washed under running water to re- move blood and then the teeth were scaled using sharp hand scaler (Martin-Germany) to remove any plaque, calculus, mucous and adhering soft tissue shreds. Teeth were examined with magnifying lens at 6x magnification (Univit) to exclude any teeth with cracks, fracture or pathological abnormality. The teeth were stored in deinoized water and thymol at refrigerator $4{ }^{\circ} \mathrm{C}$ for a maximum period of one month until used ${ }^{(9)}$.

\section{Preparation of natural cross-linkers agents:}

A. Hesperidin: $5 \mathrm{~g}$ powder was dissolved in $5 \mathrm{ml}$ $100 \%$ dimethylsulphoxide oxide (DMSO) then diluted with distilled water to be 0.5 with $\%$ to $10 \%$ DMSO as the final concentration ${ }^{(10)}$.

B.Propolis: $25 \mathrm{~g}$ powder was dissolved in $50 \mathrm{ml}$ dimethyl sulfoxide (DMSO) $(100 \%, \mathrm{w} / \mathrm{v})$ by magnetic mixer for $24 \mathrm{~h}$ at $37^{\circ} \mathrm{C}$. Working solutions at concentrations of $10 \%$ were then prepared in sterile saline solution $^{(11)}$.

\section{Specimens preparation:}

The occlusal enamel of each sound tooth was ground using a grinding machine (Demco E96, Dental Maintenance co., INC.USA.) under water coolant to expose the dentin just beneath DEJ (dentin enamel junction). Dentin surface were hand polished with 600 grit silicon carbide abrasive paper under running water to create a uniform surface. The ground surface was examined using a magnifying lens at $6 x$ magnification to verify that all enamel was removed. The flat surface was acid-etched for 15 seconds with 37\% phosphoric acid Scotchbond universal etchant) then thoroughly rinsed with water for 15 seconds and dried with air.

\section{Specimens grouping}

The specimens were divided into three main groups according to materials used for dentin surface treatment. A1: the dentin surface treated with Propolis solution for $20 \mathrm{~s}$ then dried with air for 
10s. ${ }^{(11)}$ A2: the dentin surface treated by Hesperidin at $37 \mathrm{c}$ for $12 \mathrm{~h}^{(10)}$, then dried by air A3: did not receive any treatment. Disposable applicator brush was used to apply 2 consecutive coats of the adhesive (with fully saturated brush tip) to the entire tooth structure and was rubbing it in for 20 seconds, subsequently a gentle stream of air was directed over the liquid for about 5 seconds until it no longer moved to evaporate the solvent completely. The adhesive was then curing with a 3M ESPE Eliper light curing unit (LEDition curing unit 3M ESPE AG, ESPE Platz, 82229 Seefeld. Germany.) at an intensity of $1200 \mathrm{~W} / \mathrm{cm}^{2}$ for 20 seconds. The surface was built up with resin composite (Filtek Z350XT body shade A3) by using specially constructed Teflon mold with internal dimensions of $2 \mathrm{~mm}$ height and $3 \mathrm{~mm}$ diameter ${ }^{(12)}$ then cured by $3 \mathrm{M}$ ESPE Eliper light curing unit for 20 seconds.

\section{Specimens storage:}

The specimens were further divided into three subgroups according to storage media B1. acidic solution was left for $2 \mathrm{hr}$ then washed with distilled water and stored in deionized water for the rest period $^{(13)}$. B2. artificial saliva at room temperature ranged from $\left(25 \operatorname{to}^{\circ} 0^{\circ} \mathrm{C}\right)$ for 28 days ${ }^{(14)}$. The artificial saliva was refreshed every day and the specimens were washed with distilled water. B3. distilled water for 28 days. Specimens were then prepared for shear bond strength.

\section{Shear bond strength test:}

Shear bond strength testing was done using Galdabini Quaiser 600 mechanical testing machine (Gladabini Quaiser 600, Italy with a load cell of 50 $\mathrm{kN}$ ). A specially designed upper attachment for the machine was fabricated with the same dimensions of the composite disc to fit into it. The blocks were then mounted on the lower attachment and the upper attachment held the composite disc in the testing machine. They were then tested in shear at a crosshead speed of $0.5 \mathrm{~mm} / \mathrm{min}$ until fracture. Maximum load needed to facture the bond was obtained in Newton (N). Shear bond strength was then calculated from the following equation:

$$
\text { Shear bond strength }=\frac{\text { max. Load in Newton }}{\text { Area in } \mathrm{mm}^{2}}
$$

\section{Statistical analysis:}

Two-way ANOVA test was used to study the effect of material and storage media on shear bond strength. Tukey's post-hoc test was used for pairwise comparisons between variables when ANOVA test is significant. The significance level was set at $\mathrm{P} \leq 0.05$. Statistical analysis was performed by IBM (IBM Corporation, NY, USA.) SPSS (SPSS, Inc., an IBM Company.) Statistics Version 20 for Windows.

\section{RESULTS}

\section{The effect of cross linking agents on SBS:}

The mean and standard deviation for SBS of different tested cross linking materials were presented in Table (1) and Fig (1). There was a highly significant difference between the tested materials $(\mathrm{p}<0.001)$. The results revealed that Propolis showed the statistically significantly highest mean SBS value $(26.5 \pm 4.5)$. There was no statistically significant difference between Hesperidin $(24.5 \pm 2.1)$ and control group $(23.6 \pm 2.4)$ both showed statistically significantly lower mean SBS values.

Table (1) The mean and standard deviation of SBS for different cross linking agents

\begin{tabular}{|c|c|c|c|c|c|c|}
\hline \multicolumn{2}{|c|}{ Propolis } & \multicolumn{2}{c|}{ Hesperidin } & \multicolumn{2}{c|}{ Control } & \multirow{2}{*}{ P-value } \\
\cline { 1 - 5 } Mean & SD & Mean & SD & Mean & SD & \\
\hline $26.5^{\text {A }}$ & 4.5 & $24.5^{\text {В }}$ & 2.1 & $23.6^{\text {B }}$ & 2.4 & $<0.001^{*}$ \\
\hline
\end{tabular}

*: Significant at $P \leq 0.05$, Different superscripts are statistically significantly different 


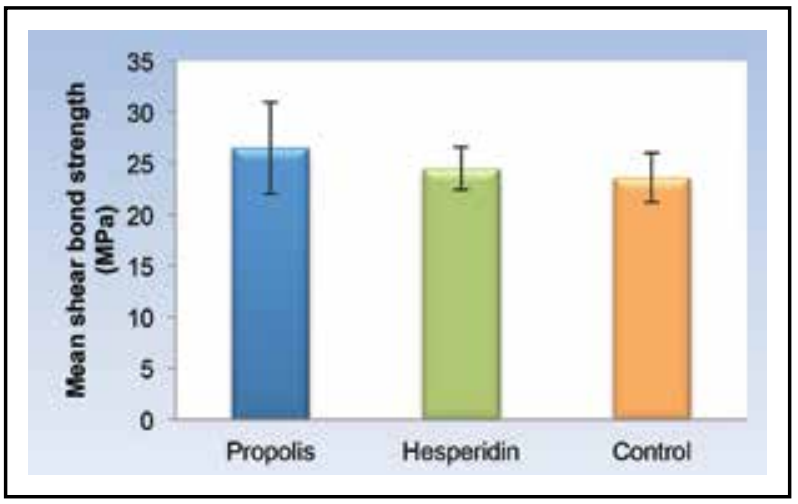

Fig. (1) Histogram representing mean and standard deviation values of shear bond strength of different cross linking agents

\section{The effect of storage media on SBS:}

The mean and standard deviation for SBS of different tested cross linking materials were presented in Table (2) and Fig (2). There was a highly significant difference between the storage media $(\mathrm{p}<0.001)$. The results revealed that distilled water showed the statistically significantly highest mean SBS value $(27.0 \pm 4.6)$. There was no statistically significant difference between acid $(23.9 \pm 1.9)$ and artificial saliva (23.8 \pm 1.8$)$; both showed a statistically significantly lower mean SBS.

Table (2) The mean and standard deviation of SBS for different storage media

\begin{tabular}{|c|c|c|c|c|c|c|}
\hline \multicolumn{2}{|c|}{ Acid } & \multicolumn{2}{c|}{$\begin{array}{c}\text { Artificial sa- } \\
\text { liva saliva }\end{array}$} & \multicolumn{2}{c|}{ Distilled water } & \multirow{2}{*}{-value } \\
\cline { 1 - 5 } Mean & SD & Mean & SD & Mean & SD & \\
\hline $23.9^{\text {в }}$ & 1.9 & $23.8^{\text {в }}$ & 1.8 & $27.0^{\text {А }}$ & 4.6 & $<0.001^{*}$ \\
\hline
\end{tabular}

*: Significant at $P \leq 0.05$, Different superscripts are statistically significantly different

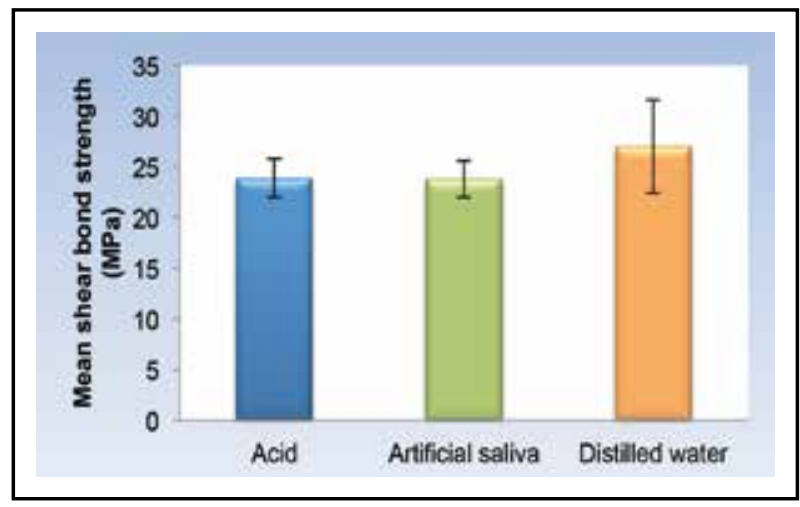

Fig. (2) Histogram representing mean and standard deviation values of shear bond strength for different storage media

\section{DISCUSSION}

Adhesive dentistry is a rapidly evolving discipline. For many years the dental profession has strived to achieve good adhesion of resin composite to tooth substrate. Increasing the collagen resistance against the degradation process may improve the stability of the resin-dentin bonded interface; this was the main purpose of incorporating collagen cross-linkers into the bonding process. Collagen cross-linkers are effective in protecting collagen fibrils from degradation through enhancing the collagen's chemical and mechanical properties. More recently their benefits in dentin bonding have been credited as they have the ability to inhibit the activity of host-derived metalloproteinases (MMPs). However, all of the cross-linking agents reduced or eliminated the MMPs' activity at the dentin-bonding interface ${ }^{(15)}$.

In the present study two cross-linkers agents were used (Propolis and Hesperidin). Propolis is a natural resinous material has been extensively used in dentistry. Flavonoids, caffic acids and cinnamic acid derivatives have been widely cited as the main biologically active compounds. However, the exact composition of Propolis from different botanical origins may vary and its biological effects are similar. Propolis being natural, anti-oxidants and free radi- 
cal scavengers, increases collagen synthesis as well as accelerates the conversion of soluble collagen to insoluble collagen during development to enhance collagen stabilization and collagen formation contributing to dentin stabilization. It can be considered as one of the pre-treatment regimes prior to application of bonding agents. This new collagen stabilizing agent seems to have a promising future to achieve a restoration with stable bonding abilities ${ }^{(6)}$.

Hesperidin (HPN) hesperetin-7-O-rutinoside is a glycoside citrus flavonoid that is able to improve the immediate bond strength, mechanical properties of resin dentin interfaces, stabilizing collagen matrix and inducing remineralization as well as management of root caries ${ }^{(16)}$. Dimethyl sulfoxide (DMSO) was used in this study to prepare Hesperidin and Propolis. It is used as solvent in adhesive dentistry due to its ability to penetrate biological surfaces without causing dissociation of extracellular matrix collagen. This effect may be related either to DMSO's ability to compete with water molecules in interpeptide hydrogen bonding in collagen matrix or the ability to change the collagen interfibril spacing on a submicrometer scale ${ }^{(17)}$.

In the present study, three storage media were used (acids, artificial saliva and distilled water) to simulate the aging phenomena within the oral cavity. The period of storage in this study was 28 days, according to the ISO technical specification 11405 which states that bond strengths should be measured within six months after teeth extraction ${ }^{(18)}$.

On comparing the mean SBS of different cross linkers, there was a statistically significant difference of SBS between the two cross linking agents. The results revealed that Propolis showed the statistically significantly highest mean shear bond strength value. This may be attributed to the presence of flavonoids in Propolis that react with collagen proteins via four mechanisms: covalent interactions, ionic interactions, hydrogen bonding and hydrophobic interactions ${ }^{(6)}$. This in turn decreases the enzymatic degradation of restoration interfaces, modifies the tooth surface with collagen cross-linking, produces fibrillar and collagen stabilization and hence increases bond strength of dentin. Moreover, Propolis stimulate the production of transforming growth factor (TGF) Beta 1 which is important for the differentiation of odontoblasts and also induces the synthesis of collagen ${ }^{(19)}$.

These results were in agreement with other studies ${ }^{(11,6)}$ which reported flavonoids and caffeic acid present in Propolis are known to play an important role in the stimulation of various enzyme systems, cell metabolism, collagen formation contributing to dentin stabilization and increase bond strength.

There was no statistically significant difference between Hesperidin and control groups; both showed statistically lower mean shear bond strength. These findings were in disagreement with a recent study ${ }^{(20)}$ which concluded that use of Hesperidin highly increases the mechanical properties this may be due to differences in the application of the material as HPN was incorporated in the primer, pretreatment agent and sample size ${ }^{(20)}$.

In this study on comparing the mean SBS of different storage media there was a statistically significant difference between different storage media. Results revealed that distilled water showed the statistically significant highest mean shear

bond strength. However, there was no statistically significant difference between acid and artificial saliva; both showed statistically significant lower mean shear bond strength. Water sorption by hydrophilic and ionic resin monomers within both hybrid layer and adhesive layer may contribute to the degradation of resin-dentin bond strength over time. Also incompletely infiltrated deep areas in the hybrid layer contain denuded collagen fibrils which could be responsible for degradation and provide a pathway for host derived enzymes. These enzymes are class of zinc and calcium dependent endopeptides and responsible for auto-degradation of collagen matrices. This might have led to hydrolytic degradation and decrease the bond strength over 
time due to thinning and disappearance of collagen fibrils from incompletely infiltrated aged a hybrid layer ${ }^{(21)}$.

The current results revealed that Propolis showed the statistically significantly higher mean shear bond strength after storage in distilled water. However, there was no statistically significant difference between acid and artificial saliva; both showed statistically significant lower mean shear bond strength. This result was in agreement with a study ${ }^{(22)}$ that found after aging, a decrease in bonding effectiveness was observed in all materials after aging. Similar results were also found by other study ${ }^{(23)}$ who demonstrated a decrease in the bond strength of total-etch adhesive system.

Regarding Hesperidin, there was no statistically significant difference between the different storage media. This result was in agreement with a recent study $^{(20)}$ who believed that Hesperidin have a potential to preserve the bonded interface, thereby improving the long-term bond durability.

\section{CONCLUSIONS}

1- Both Propolis and Hesperidin cross linking agents are considered beneficial in improving bonding to dentin, though Propolis was much more effective.

2- Storage media has deteriorating effect on resindentin shear bond.

\section{REFERENCES}

1. Islam MS, Hiraishi N, Nassar M, Yiu C, Otsuki M, Tagami J. Effect of hesperidin incorporation into a self-etching primer on durability of dentin bond. Dent Mater. 2014; 30(11):1205-12.

2. Gaengler $\mathrm{P}$, Hoyer I, Montag R, Gaebler P. Micromorphological evaluation of posterior composite restorations - a 10-year report. J oral rehab. 2004 ;31 (10): 991-1000

3. Opdam NJM, Loomans BAC, Roeters FJM, Bronkhorst EM. Five-year clinical performance of posterior resin composite restorations placed by dental students. J Dent. 2004; 32 (5): 379-83.
4. Nagpal R, Singh P, Singh S, Tyagi Sp. Proanthocuanidin: A natural dentin biomodifier in adhesive dentistry. J Res Dent 2016; (4): 1-6

5. Bedran"Russo AKB, Pashley DH, Agee K, Drummond JL, Miescke KJ. Changes in stiffness of demineralized dentin following application of collagen crosslinkers. J Biomed Mater Res. Part B: App Biomater. 2008; 86(2):330-4.

6. Suresh M, Madhubala M, Sanjeev K, Sekar M. Clotting Co-Factor and Bees Extract in Dentin Stabilization. World J of Med Sci. 2014; 10(2):204-7.

7. Strijp AJ, Takatsuka T, Sono R, Iijima Y. Inhibition of dentine collagen degradation by hesperidin: an in situ study. Eur J Oral Sci. 2015; 123(6):447-52.

8. Skovron L, Kogeo D, Gordillo LAA, Meier MM, Gomes OM, Reis A, et al. Effects of immersion time and frequency of water exchange on durability of etch"and" rinse adhesive. J Biomed Mater Res Part B: App Biomater. 2010; 95(2):339-46.

9. Salza $U$ and Bockb T. Testing adhesion of direct restoratives to dental hard tissue a review. J Adhes Dent. 2010;12(5):343-71.

10. Hiraishi N, Sono R, Sofiqul I, Yiu C, Nakamura H, Otsuki M, et al. In vitro evaluation of plant-derived agents to preserve dentin collagen. Dent Mater. 2013; 29(10):1048-54.

11. Öznurhan F, Buldur B, Ozturk C, Durer A. Cumhuriyet Dent J. 2015.18-22.

12. Almuammar M, Schulman A, Salama F. Shear bond strength of six restorative materials. J Clin Pediatr Dent. $2001 ; 25(3): 221-25$.

13. Eisenburger M, Shellis R, Addy M. Scanning electron microscopy of softened enamel. Caries research. 2003;38(1):67-74.

14. Rodrigues JA, Oliveira GPF, Amaral CM. Effect of thickener agents on dental enamel microhardness submitted to at-home bleaching. Braz Oral Res. 2007; 21(2):170-75.

15. Macedo G, Yamauchi M, Bedran-Russo A. Effects of chemical cross-linkers on caries-affected dentin bonding. J Dent Res. 2009;88(12):1096-100.

16. Hiraishi N, Sono R, Islam M, Otsuki M, Tagami J, Takatsuka T. Effect of hesperidin in vitro on root dentine collagen and demineralization. J Dent. 2011; 39(5):391-96.

17. Tjäderhane L, Mehtälä $P$, Scaffa $P$, Vidal C, Pääkkönen V, Breschi L, et al. The effect of dimethyl sulfoxide (DMSO) on dentin bonding and nanoleakage of etch-and-rinse adhesives. Dent Mater. 2013; 29(10):1055-62. 
18. Dental materials - testing of adhesion to tooth structure. Second ed. Switzerland: 2003. Technical specification ISO/TS 11405 .

19. Kim JI, Pant HR, Sim H-J, Lee KM, Kim CS. Electrospun propolis/polyurethane composite nanofibers for biomedical applications. Materials Science and Engineering: C. 2014; 44:52-7.

20. Islam S, Hiraishi N, Nassar M, Yiu C, Otsuki M, Tagami J. Effect of natural cross-linkers incorporation in a selfetching primer on dentin bond strength. J Dent. 2012; 40(12):1052-59.
21. Tay F, Pashley DH, Su B, Hiraishi N, Yiu C. Water treeing in simplified dentin a -dhesives dejavu. OPERATIVE DENTISTRY-UNIVERSITY OF WASHINGTON-. 2005; 30(5):561.

22. Makishi P, André C, Ayres A, Martins A, Giannini M. Effect of Storage Time on Bond Strength and Nanoleakage Expression of Universal Adhesives Bonded to Dentin and Etched Enamel. Oper Dent. 2016; 41(3):305-17.

23. Hashimoto M, Fujita S, Nagano F, Ohno H, Endo K. Ten" years degradation of resin-dentin bonds. Eur J Oral Sci. 2010; 118(4):404-10. 